\title{
Compression and swelling of hydrogels in polymer solutions: A dominant-mode model
}

\author{
Melle T. J. J. M. Punter $\odot,{ }^{1}$ Hans M. Wyss, ${ }^{2,3,4}$ and Bela M. Mulder ${ }^{1}$ \\ ${ }^{1}$ Institute AMOLF, Theory of Biomolecular Matter, Science Park 104, 1098XG Amsterdam, The Netherlands \\ ${ }^{2}$ Department of Mechanical Engineering, Materials Technology, Eindhoven University of Technology, 5600MB Eindhoven, The Netherlands \\ ${ }^{3}$ Institute for Complex Molecular Systems, Eindhoven University of Technology, 5600MB Eindhoven, The Netherlands \\ ${ }^{4}$ Dutch Polymer Institute, P.O. Box 902, 5600AX Eindhoven, The Netherlands
}

(Received 28 July 2020; accepted 20 October 2020; published 9 December 2020)

\begin{abstract}
The swelling and compression of hydrogels in polymer solutions can be understood by considering hydrogelosmolyte-solvent interactions which determine the osmotic pressure difference between the inside and the outside of a hydrogel particle and the changes in effective solvent quality for the hydrogel network. Using the theory of poroelasticity, we find the exact solution to hydrogel dynamics in a dilute polymer solution, which quantifies the effect of diffusion and partitioning of osmolyte and the related solvent quality change to the volumetric changes of the hydrogel network. By making a dominant-mode assumption, we propose a model for the swelling and compression dynamics of (spherical) hydrogels in concentrated polymer solutions. Osmolyte diffusion induces a biexponential response in the size of the hydrogel radius, whereas osmolyte partitioning and solvent quality effects induce monoexponential responses. Comparison of the dominant-mode model to experiments provides reasonable values for the compressive bulk modulus of a hydrogel particle, the permeability of the hydrogel network, and the diffusion constant of osmolyte molecules inside the hydrogel network. Our model shows that hydrogel-osmolyte interactions can be described in a conceptually simple manner, while still capturing the rich (de)swelling behaviors observed in experiments. We expect our approach to provide a roadmap for further research into and applications of hydrogel dynamics induced by, for example, changes in the temperature and the $p \mathrm{H}$.
\end{abstract}

DOI: 10.1103/PhysRevE.102.062607

\section{INTRODUCTION}

Hydrogels are cross-linked polymer networks which, when fully swollen, typically imbibe large volumes of water relative to their dry volume. They can have strong volumetric responses to changes in, for example, the $p \mathrm{H}$, the temperature, and the osmotic pressure of their surroundings, which makes them interesting for drug delivery and responsive materials like actuators and soft robotics [1-3].

Recently, the compression and reswelling of microgel particles in response to an osmotic shock have been reported [4]. Initially fully swollen microgels were brought into a continuously flowing polymer solution, and from both phenomenological and formative poroelastic modeling of their volumetric response the diffusion constant of osmolyte inside the hydrogels, the permeability, and the bulk modulus of the hydrogel network could be inferred. Due to nonlinearities, both the phenomenological and poroelastic model were solved numerically.

Moreover, remarkable overshoot behavior of hydrogels swelling in polymer solutions has been reported. These

Published by the American Physical Society under the terms of the Creative Commons Attribution 4.0 International license. Further distribution of this work must maintain attribution to the author(s) and the published article's title, journal citation, and DOI. experiments have been described using a numerically solved relaxational dynamics model based on the free energy of a hydrogel particle, but this model provides little direct insight into the resulting underlying hydrogel dynamics [5]. To obtain a more fundamental understanding of the physical mechanisms that govern these (de)swelling processes, an analytical model description, where the role of the different physical properties is more directly evident, would be desirable.

Here, using the theory of poromechanics, we propose a dominant-mode model describing the volumetric response of hydrogels for both swelling and compression in concentrated polymer solutions. Large volumetric changes of hydrogels typically give rise to nonlinearities in the compressive bulk modulus of the hydrogel, the permeability of the hydrogel network, and the diffusion coefficient of the osmolyte. To obtain explicit insight into the hydrogel dynamics, we solve the osmolyte mass conservation equation and the force balance equation exactly for hydrogels in dilute polymer solutions. We find the temporal effect of osmolyte diffusion on the hydrogel radius to be of biexponential form, expressing the interplay between osmolytes that stresses the hydrogel network and the resulting "diffusion" of the hydrogel network [6,7]. Also, partitioning of the osmolyte between the hydrogel and the surrounding polymer solution, and changes in the quality of the solvent for the hydrogel network, induce monoexponential swelling or compression to the equilibrium state, determined solely by hydrogel network diffusion. From this exact solution we approximate the hydrogel response to 
concentrated polymer solutions by making a dominant-mode assumption.

The dominant-mode model is fitted to the measured volumetric response of hydrogels from both Sleeboom et al. [4] and Aangenendt et al. [5]. Reasonable values are obtained for the (effective) diffusion constant, permeability, and bulk modulus, which scale as expected with the concentration of the hydrogel network and osmolyte. The dominant-mode model provides fit values for the material parameters consistent with expected trends.

After briefly introducing the swelling and compression experiments on hydrogel particles we reanalyze here, the equations of motion of hydrogel dynamics are formulated using the theory of poroelasticity. With the exact solution of hydrogel-osmolyte dynamics in a dilute polymer solution, we formulate the dominant-mode model. Finally, the model is compared to the experiments, and the estimations for the compressive modulus, the hydrogel permeability, and the osmolyte diffusion constant are discussed.

\section{SWELLING AND COMPRESSION EXPERIMENTS}

\section{A. Microgels}

In the experiments of Sleeboom et al. [4] microgels with various acrylamide monomer concentrations and crosslinker densities were synthesized. This resulted in "soft," "medium," and "stiff" microgel particles, depending on their estimated compressive modulus, as assessed with capillary micromechanics (CM) [8]. The microgels were trapped in a microfluidic device where they were first equilibrated in demi-water and subsequently compressed by replacing the demi-water by a continuously flowing polymer solution of dextran 70k (from Leuconostoc spp, $M_{w}=70 \mathrm{kDa}$, Sigma-Aldrich, radius of gyration $R_{g} \approx 6 \mathrm{~nm}$ [9]) [see Figs. 1(a) and 1(b)]. The hydrogels initially decrease in volume and either reswell to (approximately) their original volume or deswell to some reduced volume [see Figs. 1(c)1(f)]. The experiments in Figs. 1(d)-1(f) show that a higher osmotic pressure induces a faster initial decrease in hydrogel volume, but [see Fig. 1(f)] not necessarily a smaller equilibrium volume. Therefore, apart from the osmotic pressure, solvent-quality effects are expected to be relevant for the determination of the equilibrium volume.

\section{B. Aquapearls}

In the experiments of Aangenendt et al. [5] so-called aquapearls (sodium polyacrylate particles) were used as model hydrogel particles of macroscopic size. Swelling experiments were conducted by having dried hydrogel particles swell in polymer solutions of dextran $70 \mathrm{k}$, polyethylene glycol 20k (PEG 20k, $M_{w}=20 \mathrm{kDa}$, Sigma-Aldrich, $R_{g} \approx$ $7 \mathrm{~nm}$ [10]) and polyethylene oxide 200k (PEO 200k, $M_{w}=$ $200 \mathrm{kDa}$, Sigma-Aldrich, $R_{g} \approx 22 \mathrm{~nm} \mathrm{[10])} \mathrm{at} \mathrm{various} \mathrm{os-}$ molyte concentrations. It was shown that the combination of alterations in solvent quality and the osmotic pressure differences due to partitioning of osmolyte between the polymer solution and the hydrogel particle could explain
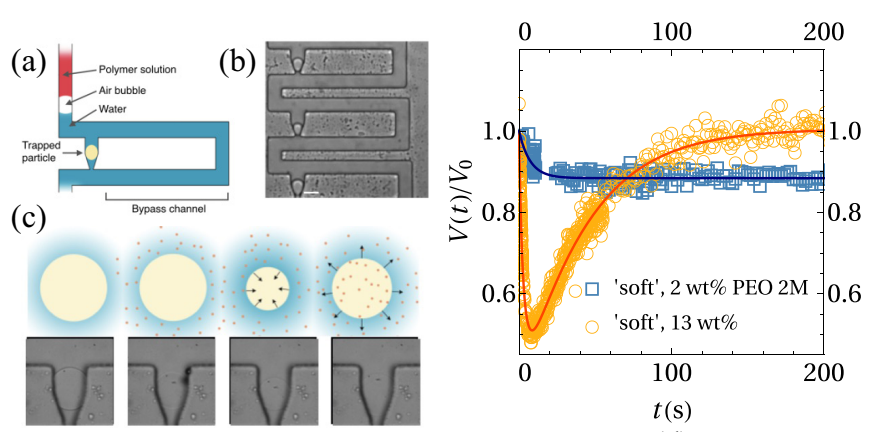

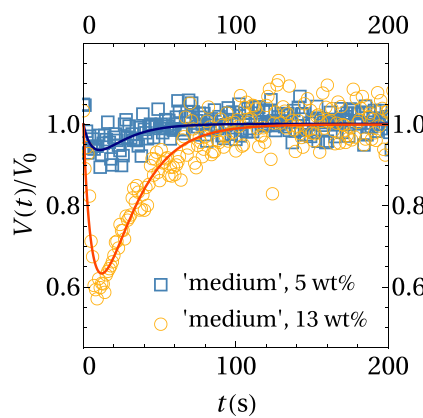

(e)

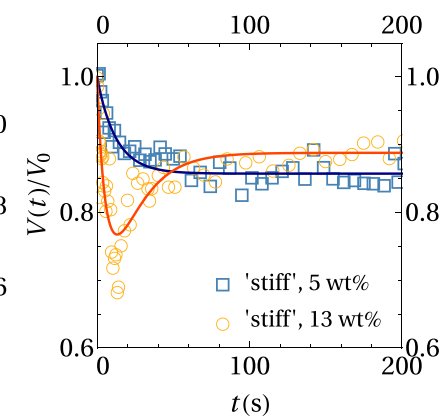

(f)
FIG. 1. Schematic (a) and microscopy (b) image of the microfluidic setup in which microgels, with radius $a=20 \mu \mathrm{m}$, are trapped and the surrounding water (blue) can be replaced by a polymer solution (red). The ratio of the volume $V(t)$ of soft (d), medium (e), and stiff (f) microgels to their original volume $V_{0}$ is given as a function of time $t$ after water is replaced by a dextran 70k polymer solution, unless specified otherwise. Replacing water by a PEO 2M polymer solution (d) shows that a large osmolyte causes permanent compression of the hydrogel particle. The solid lines are fits to the dominant-mode model, with parameters displayed in Table I. [Panels (a)-(c) are adapted from Sleeboom et al. [4].]

the observed overshoot in the PEO 200k swelling experiments and the monotonic swelling observed in dextran $70 \mathrm{k}$ and PEG 20k swelling experiments (see Fig. 2). Likewise, solvent quality and partitioning could account for the undershoot and monotonic deswelling observed in compression experiments (see Fig. 3).

\section{POROELASTIC MODELING}

We first outline the basic equations of poroelasticity $[7,11,12]$, from which the exact solution for a hydrogel particle in a dilute polymer solution is found. Assuming the long-time dynamics of this solution to be similar to those in the regime of concentrated polymer solutions, we construct a dominant-mode model for hydrogel swelling and compression.

\section{A. Hydrogel dynamics in dilute polymer solutions}

Regarding the hydrogel particle as a superposed solid phase (hydrogel network) and fluid phase (dilute polymer solution), and the osmolyte particles as a constituent of the 
$\nabla \mathrm{NaCl} \approx 2 \mathrm{wt} \% \mathrm{PEO} 200 \mathrm{k} \circ 4 \mathrm{wt} \%$ PEO 200k $\quad 5 \mathrm{wt} \%$ PEO 200k

$\triangle 7.5 \mathrm{wt} \%$ PEO $200 \mathrm{k} \triangle 10 \mathrm{wt} \%$ PEO $200 \mathrm{k}$

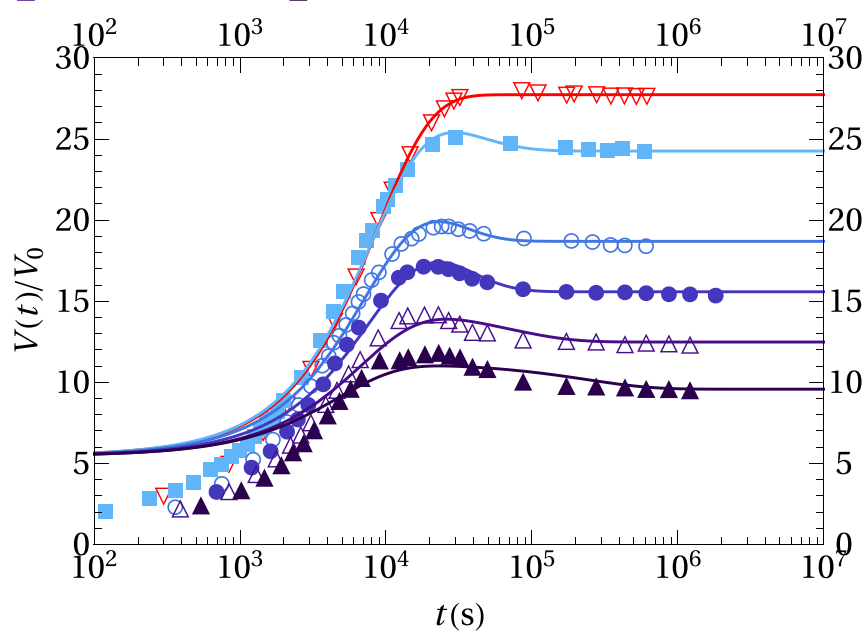

(a)

$\nabla \mathrm{NaCl} \quad \square 8 \mathrm{wt} \%$ dextran $70 \mathrm{k} \bigcirc 8 \mathrm{wt} \%$ PEG 20k

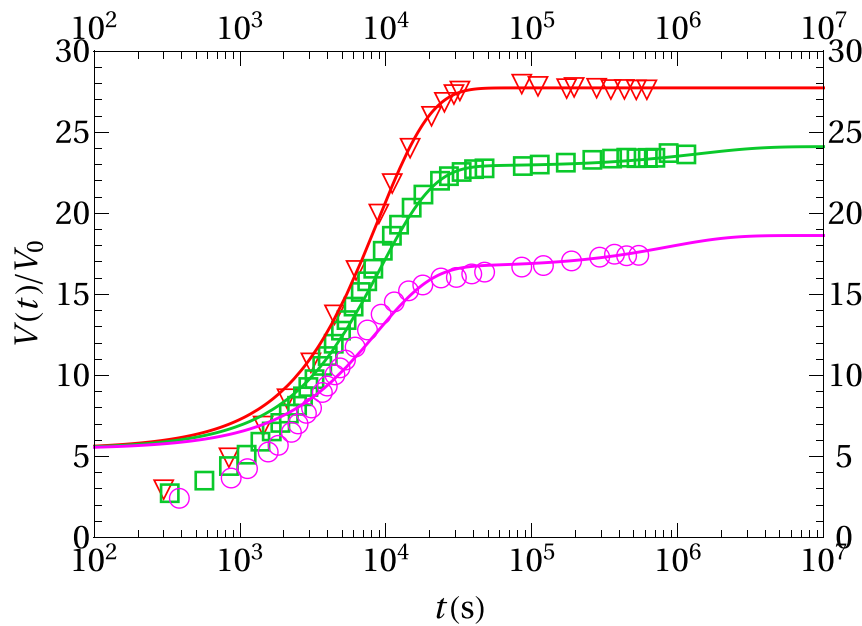

(b)

FIG. 2. The normalized volume $V(t) / V_{0}$ as a function of time $t$ of initially dry aquapearl hydrogel particles for different PEO $200 \mathrm{k}$ concentrations exhibiting an overshoot (a), and for dextran 70k and PEG 20k exhibiting no overshoot (b). The solid lines are fits of the dominant-mode model, with parameters displayed in the upper half of Table II.

fluid phase $[13,14]$, the mass conservation equations read in Eulerian form

$$
\begin{gathered}
\frac{\partial \phi_{\mathrm{s}}}{\partial t}+\nabla \cdot\left(\phi_{\mathrm{s}} \mathbf{v}_{\mathrm{s}}\right)=0 \\
\frac{\partial \phi_{\mathrm{n}}}{\partial t}+\nabla \cdot\left(\phi_{\mathrm{n}} \mathbf{v}_{\mathrm{n}}\right)=0 \\
\frac{\partial c \phi_{\mathrm{s}}}{\partial t}+\nabla \cdot\left[c \phi_{\mathrm{s}}\left(\mathbf{v}_{\mathrm{n}}+\mathbf{v}_{\text {dif }}\right)\right]=0
\end{gathered}
$$

where both phases are assumed to be incompressible; $\phi_{\mathrm{s}}$ and $\phi_{\mathrm{n}}$ are, respectively, the volume fractions of the polymer solution and the hydrogel network; $\mathbf{v}_{\mathrm{s}}$ and $\mathbf{v}_{\mathrm{n}}$ are their respective velocities; and $c$ is the concentration of osmolyte particles per

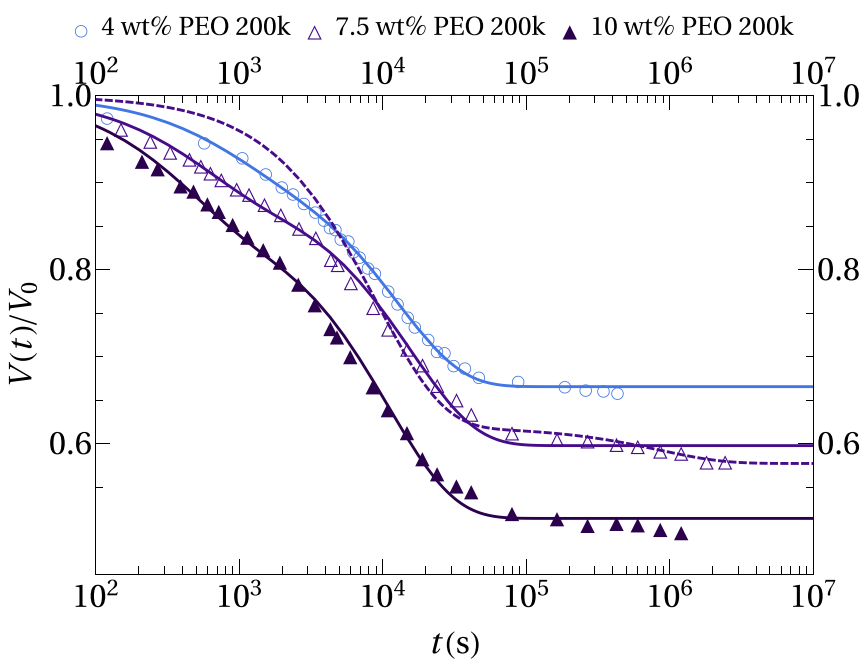

(a)

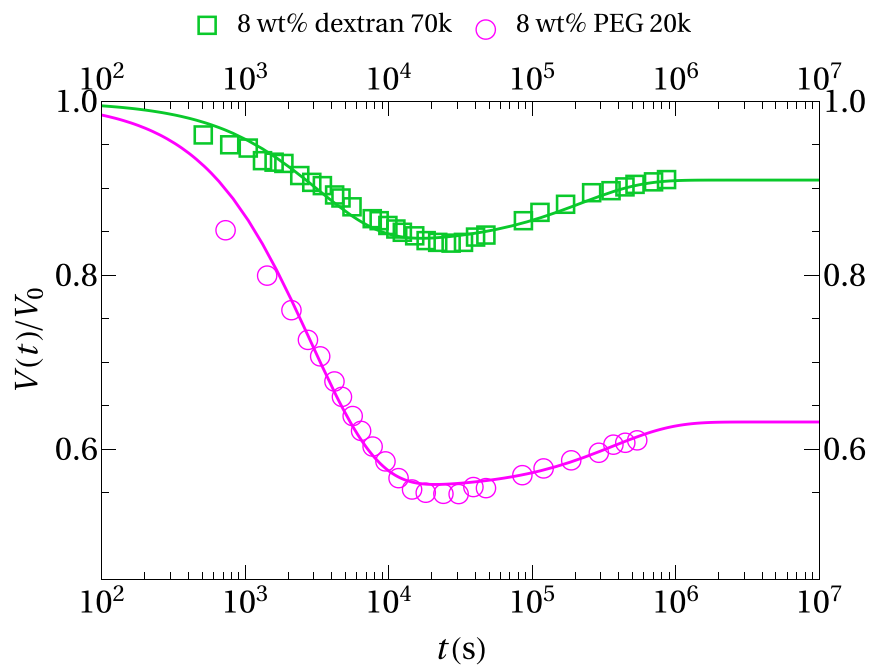

(b)

FIG. 3. The normalized volume $V(t) / V_{0}$ as a function of time $t$ of aquapearl hydrogel particles in various PEO 200k concentrations, exhibiting no undershoot (a), and for dextran 70k and PEG 20k exhibiting an undershoot (b). The solid lines are fits to the dominantmode model, with parameters displayed in the lower half of Table II. The dashed line is a fit of the 7.5-wt-\% PEO 200k experiment which is forced to take into account the final stage of slow deswelling (see Table III for fit parameters).

unit volume of polymer solution. We idealize the hydrogel particle as being spherically symmetric. Summing Eqs. (1) and (2), using that $\phi_{\mathrm{s}}+\phi_{\mathrm{n}}=1$, and applying the divergence theorem, we find

$$
\phi_{\mathrm{s}} \mathbf{v}_{\mathrm{s}}+\phi_{\mathrm{n}} \mathbf{v}_{\mathrm{n}}=0
$$

which directly relates the velocity of the polymer solution to the velocity of the hydrogel network.

The advective osmolyte velocity in Eq. (3) equals $\mathbf{v}_{\mathrm{n}}$, because the osmolyte particles are assumed to have much stronger frictional interaction with the hydrogel network than with the solvent, i.e., the hydrogel network and the osmolyte particles move advectively as a single complex. On the other hand, in order for the osmolyte to enter the hydrogel 
diffusively on experimentally relevant time scales it should not be so large that the much slower reptation mechanism becomes dominant. We therefore assume that the radius of gyration of an osmolyte particle $R_{\mathrm{g}}$ is comparable to the mesh size $\xi$ of the hydrogel network, i.e., $R_{\mathrm{g}} \sim \xi$.

Assuming osmolyte diffusion to be Fickian, the velocity of osmolyte particles relative to the hydrogel network $\mathbf{v}_{\text {dif }}$ is given as

$$
c \mathbf{v}_{\text {dif }}=-D_{\text {osm }} \nabla c,
$$

with $D_{\text {osm }}$ the diffusion coefficient of osmolyte inside the hydrogel network.

Assuming mechanical equilibrium and the absence of body forces, the overall force balance for the hydrogel can be written as

$$
\nabla \cdot \sigma=0
$$

with $\sigma$ the Cauchy stress tensor of the whole hydrogel particle, i.e., of the polymer solution and hydrogel network combined. Using the Terzaghi effective stress [12,15], we can decompose $\sigma$ as

$$
\sigma_{i j}=\sigma_{i j}^{\prime}-(p+\Pi) \delta_{i j}
$$

where $\sigma^{\prime}$ is the elastic stress of the hydrogel network, and $p$, $\Pi$, and $p+\Pi$ are, respectively, the pneumatic pressure, the total osmotic pressure, and the total pressure of the polymer solution [16]. For a dilute polymer solution in the hydrogel network, the total osmotic pressure $\Pi=\Pi_{\text {id }}+\Pi_{\text {exc }}$ is composed of the ideal part $\Pi_{\mathrm{id}}=k_{\mathrm{B}} T c$ and the excess pressure due to hydrogel network-osmolyte interactions $\Pi_{\mathrm{exc}}=$ $k_{\mathrm{B}} T c \rho B_{\mathrm{ns}}$, where $\rho$ is the number density of the hydrogel network and $B_{\mathrm{ns}}$ is the cross-virial coefficient of interactions between the hydrogel network and the osmolyte in the polymer solution, through which the solvent quality can be increased, $\Pi_{\text {exc }}>0$, or decreased, $\Pi_{\text {exc }}<0$. Darcy's law relates gradients in the pneumatic pressure to the flow of solvent through the hydrogel-osmolyte complex as [12]

$$
\phi_{\mathrm{s}}\left(\mathbf{v}_{\mathrm{s}}-\mathbf{v}_{\mathrm{n}}\right)=-\frac{\kappa}{\eta} \nabla p,
$$

where $\kappa$ is the permeability of the hydrogel-osmolyte complex for the solvent, and $\eta$ is the dynamic viscosity of the solvent.

For dilute polymer solutions, the total osmotic pressure is low, implying the strain in the hydrogel network to be small, and thus the network responds approximately as a linear elastic material. Assuming the hydrogel network to be isotropic and homogeneous, the elastic stress of the hydrogel network, relative to the stress-free equilibrium state in osmolyte-free solvent, is written as

$$
\sigma_{i j}^{\prime}=\frac{M}{1-v}\left[v e_{k k} \delta_{i j}+\left(\frac{1}{2}-v\right) e_{i j}\right],
$$

with $e_{i j}=\frac{1}{2}\left(\nabla_{i} U_{j}+\nabla_{j} U_{i}\right)$ the infinitesimal strain tensor, $M=K+4 G / 3$ the longitudinal modulus of the hydrogel network [17], $K$ the compressive bulk modulus, $G$ the shear modulus, and $v$ the Poisson ratio. The displacement field $\mathbf{U}(r, t)=U(r, t) \hat{r}$ is radial, with $r \in\left[0, R_{0}\right]$ the radial position in the network and $R_{0}$ the stress-free hydrogel radius.
Mass conservation of osmolyte [Eq. (3)] and the overall force balance [Eq. (6)] are found as

$$
\begin{gathered}
\frac{\partial c}{\partial t}-D_{\mathrm{osm}} \frac{1}{r^{2}} \frac{\partial}{\partial r} r^{2} \frac{\partial c}{\partial r}=0 \\
\frac{\eta}{\kappa} \frac{\partial U}{\partial t}-M \frac{\partial}{\partial r} \frac{1}{r^{2}} \frac{\partial}{\partial r} r^{2} U=-\frac{\partial}{\partial r} k_{\mathrm{B}} T c(1+f),
\end{gathered}
$$

where we defined $f \equiv \rho B_{\text {ns }}$. Equation (10) is the standard diffusion equation for osmolyte in the hydrogel network. The displacement field [see Eq. (11)] obeys a diffusionlike equation sourced by the gradient in total osmotic pressure. These equations can alternatively be derived from the framework of incompressible mixtures [14].

Due to both short-range repulsive and long-range interactions with the hydrogel network, osmolyte is partitioned between the hydrogel particle and the surrounding polymer solution. The equilibrium partition coefficient $Q$ gives the ratio of the equilibrium concentration of osmolyte in the hydrogel to the osmolyte concentration in the surrounding medium [18]. As the hydrogel network is homogeneous, the osmolyte concentration at the hydrogel surface equals the equilibrium concentration for all times:

$$
c(r, t)=Q c_{0}, \quad \text { at } r=R_{0},
$$

with $c_{0}$ the concentration of osmolyte in the surrounding polymer solution. This concentration can be considered constant, because experimentally the microgel dynamics were shown to be independent of the velocity of the polymer solution [4]. Similarly, the aquapearl particles were put on a roller bank in a bath of polymer solution to ensure a constant outside osmolyte concentration [5]. Initially, no osmolyte is present in the hydrogel:

$$
c(r, t)=0, \quad \text { at } t=0 .
$$

Force balance requires the total pressure of the surrounding polymer solution to equal the total radial stress at the hydrogel surface, i.e., $\sigma_{r r}\left(R_{0}, t\right)=-\left(p_{0}+\Pi_{0}\right)$, with $\sigma_{r r}$ given by Eq. (7), $p_{0}$ the pneumatic pressure of the outside polymer solution, and $\Pi_{0}=k_{\mathrm{B}} T c_{0}$ the osmotic pressure of the outside polymer solution. Using that $\Pi=k_{\mathrm{B}} T c(1+f)$, invoking Eq. (12), and using the fact that the hydrogel network is permeable for solvent $p\left(R_{0}, t\right)=p_{0}$, the radial network stress at the hydrogel surface is given as

$$
\sigma_{r r}^{\prime}(r, t)=-k_{\mathrm{B}} T c_{0}[1-Q(1+f)], \quad \text { at } r=R_{0},
$$

which shows that the difference in osmotic pressure between the surface of the hydrogel and the surrounding polymer solution can provide a resultant force on the hydrogel surface. In a swelling experiment, where the hydrogel network swells from its fully dried state, the network is initially compressed relative to its stress-free state. In a compression experiment, on the other hand, the hydrogel particle starts out in the stressfree state, giving as initial condition

$$
U(r, t)=r\left(\frac{R_{\text {init }}}{R_{0}}-1\right), \quad \text { at } t=0,
$$

where $R_{\text {init }}$ is the initial radius of the hydrogel network and $R_{0}$ is the osmolyte-free equilibrium radius. 
Equations (12)-(15) provide the necessary boundary conditions to solve Eqs. (10) and (11). The solution to Eq. (10) can be written, using a Hankel transform [19], as

$$
\frac{c(r, t)}{Q c_{0}}=1-\sum_{k=1}^{\infty} 2(-1)^{k+1} j_{0}\left(\pi k \frac{r}{R_{0}}\right) \exp \left(-(\pi)^{2} \frac{t}{\tau_{\mathrm{osm}}}\right),
$$

where $j_{0}$ is the spherical Bessel function of the first kind of order zero, and $\tau_{\text {osm }} \equiv R_{0}^{2} / D_{\text {osm }}$ is the time for the osmolyte to diffuse completely through the hydrogel. Next, we may solve Eq. (11) using another Hankel transform to find

$$
\begin{gathered}
U(r, t)=\sum_{m=1}^{\infty} \frac{U_{m}(t)}{\beta_{m}^{2} N_{m}} j_{1}\left(\beta_{m} r / R_{0}\right), \\
U_{m}(t)=U_{m}^{\mathrm{init}}(t)+U_{m}^{\mathrm{bc}}(t)+\sum_{k=1}^{\infty} U_{k m}^{\mathrm{dif}}(t),
\end{gathered}
$$

where $\beta_{m}$ is the $m$ th positive solution of

$$
2 \frac{1-2 v}{1-v} j_{1}\left(\beta_{m}\right)=\beta_{m} j_{0}\left(\beta_{m}\right)
$$

and $N_{m}$ is the normalization of the $m$ th mode of the network displacement field:

$$
N_{m}=\frac{1}{2 \beta_{m}^{4}}\left(\cos \left(2 \beta_{m}\right)-1+\frac{\beta_{m}}{2} \sin \left(2 \beta_{m}\right)+\beta_{m}^{2}\right) .
$$

The effect of swelling from an initially compressed or swollen state is given as

$$
\frac{U_{m}^{\mathrm{init}}(t)}{R_{0}}=\frac{R_{\text {init }} / R_{0}-1}{\left[3 j_{1}\left(\beta_{m}\right)-\beta_{m} j_{0}\left(\beta_{m}\right)\right]^{-1}} \exp \left(-\beta_{m}^{2} \frac{t}{\tau_{\mathrm{n}}}\right),
$$

where $\tau_{\mathrm{n}} \equiv R_{0}^{2} \eta / \kappa M$ is the typical time for the hydrogel network to swell or compress. The effect of force balance at the hydrogel surface is

$$
\frac{U_{m}^{\mathrm{bc}}(t)}{R_{0}}=-\frac{[1-(1+f) Q] \Pi_{0}}{M} \frac{1-\exp \left(-\beta_{m}^{2} t / \tau_{\mathrm{n}}\right)}{\left[j_{1}\left(\beta_{m}\right)\right]^{-1}},
$$

and the contribution of the $k$ th mode of osmolyte diffusion to the $m$ th displacement mode is given as

$$
\frac{U_{k m}^{\mathrm{dif}}(t)}{R_{0}}=-(1+f) Q \frac{\Pi_{0}}{M} \frac{2 \sin \left(\beta_{m}\right)}{(\pi k)^{2}-\beta_{m}^{2}} \Gamma_{k m}(t),
$$

where

$$
\Gamma_{k m}(t)=\frac{\exp \left[-(\pi k)^{2} t / \tau_{\mathrm{osm}}\right]-\exp \left(-\beta_{m}^{2} t / \tau_{\mathrm{n}}\right)}{1-(\pi k)^{2} \tau_{\mathrm{n}} / \beta_{m}^{2} \tau_{\mathrm{osm}}} .
$$

Equation (21) shows that the effect of swelling from a prestressed state is determined by the hydrogel network diffusion time. Changes in the equilibrium volume due to partitioning of osmolyte and changes in solvent quality [see Eq. (22)] are also mediated on the network diffusion time scale. The effect of osmolyte diffusion in the hydrogel network [see Eqs. (23) and (24)] generates biexponential temporal effects, because the gradient of the osmotic pressure in the hydrogel network, varying on the time scale of osmolyte diffusion $\tau_{\text {osm }}$, causes stress gradients in the network.

\section{B. Dominant-mode model}

Given the dynamics for hydrogel particles in dilute polymer solutions, we construct a dominant-mode model for hydrogel dynamics in concentrated polymer solutions from the following considerations.

If the time scale of network diffusion is either smaller than or comparable to that of osmolyte diffusion, i.e., $\tau_{\mathrm{n}} \lesssim \tau_{\text {osm }}$, the first mode in Eqs. (16)-(24), with $k=m=1$, is dominant on time scale $\tau_{\text {osm }}$ in the long-time limit; all other modes decay much faster. Thus, the first mode is sufficient to understand the long-time effects of the interplay between osmolyte diffusion and hydrogel (de)swelling.

For a concentrated polymer solution, compared to a dilute polymer solution, the volume change in the hydrogel is expected to be large, because of the high osmotic pressure of the polymer solution, implying that the permeability $\kappa$, the compressive bulk modulus $K$, and the osmolyte diffusion coefficient $D_{\text {osm }}$ may vary appreciably over time. We do not expect them to vary over an order of magnitude, however, because if the hydrogel volume decreases, for example, by $75 \%$ the permeability is expected to decrease by $60 \%$, as approximately $V(t) / V_{\text {init }}=\left[\xi(t) / \xi_{\text {init }}\right]^{3}$ and $\kappa(t) \propto \xi^{2}(t)$, with $\xi(t)$ the time-varying average mesh size of the hydrogel network. The permeability is usually determined with relative uncertainties on the order of $10 \%$ [5], and similar uncertainties hold for the compressive bulk modulus [4] and the osmolyte diffusion coefficient [20]. Therefore, we assume that the variance of the material parameters does not give rise to qualitatively different hydrogel-osmolyte dynamics. This does not necessarily imply that the dilute-solution hydrogel dynamics suffices to describe the hydrogel dynamics in concentrated polymer solutions, however, because for concentrated polymer solutions the osmolyte must be described as an independent phase instead of a constituent of the fluid phase.

As a closed-form approximation to the hydrogel dynamics in concentrated polymer solutions, we propose the dominant mode of the exact solution [Eqs. (16)-(24)], but now regarding $K, \kappa$, and $D_{\text {osm }}$ as effective constants. Using only the dominant mode amounts to blurring of the hydrogel boundary, because for $t \rightarrow 0$ the gradient in the osmolyte concentration at the hydrogel surface remains finite, contrary to what Eqs. (12) and (13) imply.

For a dilute polymer solution, the total osmotic pressure of the polymer solution is the sum of the ideal contribution, which equals the osmotic pressure of the surrounding polymer solution, and an excess contribution due to osmolyte-network interactions. Therefore, in the dominant-mode model we replace the total osmotic pressure in the dominant mode of Eqs. (17)-(23) by the sum of the osmotic pressure of the surrounding concentrated polymer solution $\Pi_{0}$ and a pressure quantifying the solvent-quality change $\Pi_{\text {exc }}$, giving the radius of the hydrogel relative to the stress-free state $\Delta R(t)$ as

$$
\Delta R(t)=\Delta R^{\mathrm{init}}(t)+\Delta R^{\mathrm{bc}}(t)+\Delta R^{\mathrm{dif}}(t),
$$

with the contribution due to initial compression or swelling given by

$$
\Delta R^{\mathrm{init}}(t)=\frac{k}{\eta} \frac{M\left(1-R_{\mathrm{init}} / R_{0}\right)}{R_{0}} C^{\mathrm{init}}(v) \frac{\exp \left(-t / t_{\mathrm{n}}\right)}{-1 / t_{\mathrm{n}}},
$$


where $t_{\mathrm{n}} \equiv R_{0}^{2} \eta / \kappa M \beta_{1}^{2}$ is the exponential relaxation time for hydrogel swelling or compression. Force balance at the hydrogel surface provides

$$
\Delta R^{\mathrm{bc}}(t)=-\frac{k}{\eta} \frac{(1-Q) \Pi_{0}-Q \Pi_{\mathrm{exc}}}{R_{0}} C^{\mathrm{bc}}(v) \frac{1-\exp \left(-t / t_{\mathrm{n}}\right)}{1 / t_{\mathrm{n}}},
$$

which determines the equilibrium volume of the hydrogel immersed in polymer solution. The effect of osmolyte diffusion results in

$$
\Delta R^{\mathrm{dif}}(t)=-\frac{k}{\eta} \frac{Q\left(\Pi_{0}+\Pi_{\mathrm{exc}}\right)}{R_{0}} C^{\mathrm{dif}}(v) \frac{e^{-t / t_{\mathrm{osm}}}-e^{-t / t_{\mathrm{n}}}}{1 / t_{\mathrm{n}}-1 / t_{\mathrm{osm}}},
$$

which is proportional to the maximum osmotic pressure of the polymer solution in the hydrogel network and where $t_{\mathrm{osm}} \equiv$ $R_{0}^{2} / D_{\mathrm{osm}} \pi^{2}$ is the exponential relaxation time for osmolyte diffusion.

The proportionality constants in Eqs. (26)-(28) are given as $C^{\text {init }}(v)=j_{1}\left(\beta_{1}\right)\left[3 j_{1}\left(\beta_{1}\right)-\beta_{1} j_{0}\left(\beta_{1}\right)\right] / N_{1}, C^{\text {bc }}(v)=$ $j_{1}^{2}\left(\beta_{1}\right) / N_{1}$, and $C^{\mathrm{dif}}(\nu)=2 j_{1}\left(\beta_{1}\right) \sin \left(\beta_{1}\right) / N_{1}\left(\pi^{2}-\beta_{1}^{2}\right)$. For $-0.08<v \leqslant 1 / 2$, we can approximate $\beta_{1}$ as

$$
\beta_{1}=\pi\left(1-\frac{2(1-2 v)}{\pi^{2}-4+v\left(\pi^{2}+8\right)}\right)
$$

within an accuracy of $1 \%$. To first order in $x \equiv 1-\beta_{1} / \pi$ the proportionality constants read $C^{\text {init }}(v) \approx 6+\left(18-2 \pi^{2}\right) x$, $C^{\text {bc }}(v) \approx 2+6 x$, and $C^{\text {dif }}(v) \approx 2+3 x$, a particularly useful approximation for polyacrylamide hydrogels having a Poisson ratio of about 0.48 [21].

Prestress in the hydrogel network [see Eq. (26)] and the force balance at the hydrogel boundary [see Eq. (27)] cause swelling or compression on time scale $t_{\mathrm{n}}$ to the equilibrium state of the hydrogel, which is determined by the balance between the resultant compressive force $(1-Q) \Pi_{0}$ over the hydrogel surface and the solvent quality pressure $Q \Pi_{\mathrm{exc}}$ at the hydrogel surface. For $Q=0$ the hydrogel is compressed in equilibrium relative to the stress-free state due to the osmotic pressure of the surrounding polymer solution $\Pi_{0}>0$. If the solvent quality increases, however, such that $\Pi_{0}(1-Q)<$ $Q \Pi_{\text {exc }}$, the hydrogel can swell relative to the stress-free state.

In case osmolyte diffusion is slow, i.e., $t_{\mathrm{osm}} \gtrsim t_{\mathrm{n}}$, additional (de)swelling can occur on time scale $t_{\mathrm{osm}}$, depending on the sign of $\Pi_{0}+\Pi_{\text {exc }}$ [see Eq. (28)]. This slow (de)swelling stems from two competing effects due to osmolyte diffusion. On the one hand, the gradient in the osmotic pressure $\Pi_{0}$ vanishes, which decreases the compressive force on the hydrogel particle, thus inducing swelling. On the other hand, the gradient in solvent quality pressure $\Pi_{\mathrm{exc}}$ vanishes. The latter induces either swelling if the osmolyte increases the solvent quality, $\Pi_{\mathrm{exc}}>0$, or compression if the solvent quality is decreased, $\Pi_{\mathrm{exc}}<0$. Therefore, for a strong decrease in solvent quality $\Pi_{0}+\Pi_{\text {exc }}<0$, an overshoot in a swelling experiment can occur (see Fig. 2). Similarly, if $\Pi_{0}+\Pi_{\mathrm{exc}}>0$, an undershoot in a compression experiment is possible (see Fig. 3).

The velocity of the hydrogel surface at $t=0, v_{0}$, is given by a global form of Darcy's law $v_{0} \propto \kappa \Delta P / \eta R_{0}$, which stems from the approximative nature of the dominant-mode model. The pressure difference $\Delta P$ in Eq. (26) is proportional to the stress initially present in the hydrogel network, in Eq. (27) it is proportional to the osmotic pressure difference between the
TABLE I. Material properties based on the model fits displayed in Fig. 1. The standard error for each derived material parameter is given in brackets. We assumed all fitted parameters to be uncorrelated.

\begin{tabular}{lcccc}
\hline \hline Solution & $\begin{array}{c}K \\
(\mathrm{kPa})\end{array}$ & $\begin{array}{c}\kappa \\
\left(\mathrm{nm}^{2}\right)\end{array}$ & $\begin{array}{c}D_{\mathrm{osm}} \\
\left(\mu \mathrm{m}^{2} / \mathrm{s}\right)\end{array}$ & $\begin{array}{c}\Pi_{\text {exc }} \\
(\mathrm{kPa})\end{array}$ \\
\hline Medium 13 wt\% & $8.5(1.7)$ & $0.22(0.02)$ & $5.4(1.0)$ & $238.0(0.4)$ \\
Medium 13 wt\% & $6.3(1.1)$ & $0.25(0.03)$ & $7.1(1.0)$ & $234.0(0.8)$ \\
Medium 5 wt\% & $8.5(0)$ & $0.26(0.04)$ & $5.8(0.6)$ & $34.4(0.2)$ \\
Medium 5 wt\% & $8.5(0)$ & $0.15(0.02)$ & $4.8(0.4)$ & $-34.4(0.2)$ \\
Soft 13 wt\% & $2.0(0.1)$ & $0.53(0.01)$ & $12.7(0.3)$ & $238.0(0.1)$ \\
Soft PEO 2M & $9.1(0.1)$ & $0.58(0.04)$ & $0.0(0)$ & $0.0(0)$ \\
Stiff 5 wt\% & $17.0(0)$ & $0.18(0.11)$ & $7.5(293.0)$ & $3.0(4.8)$ \\
Stiff 13 wt\% & $17.0(0)$ & $0.13(0.01)$ & $5.9(0.6)$ & $1280.0(10.8)$ \\
\hline \hline
\end{tabular}

inside and the outside of the hydrogel in equilibrium, and in Eq. (28) it is proportional to the equilibrium osmotic pressure of the polymer solution in the hydrogel network.

To test the validity of the dominant-mode model, we compare it to measurements of the hydrogel volume in the swelling and compression experiments described in Sec. II.

\section{COMPARISON TO EXPERIMENT}

To test the dominant-mode model proposed in the previous section for validity, we use Eqs. (25)-(28) to obtain estimates for the (effective) osmolyte diffusion constant $D_{\text {osm }}$, and both the compressive bulk modulus $K \equiv M(1+v) / 3(1-v)$ and the permeability $\kappa$ of the hydrogel particles, with $M$ the longitudinal modulus and $v \approx 0.48$ the Poisson ratio [21,22], by fitting the dominant-mode model to the measured volumetric response of both the microgels and the aquapearl hydrogels (see Figs. 1-3). Also, we find an estimation for the change in solvent quality as the excess osmotic pressure $\Pi_{\mathrm{exc}}$ of the osmolyte in the hydrogel network. We estimate the equilibrium partition coefficient $Q$ either from literature for the microgels [23] or by modeling the available volume in the hydrogel network as spherical pores for the aquapearls (see Appendix F of Aangenendt et al. [5]). The osmotic pressures of the polymer solutions $\Pi_{0}$ as a function of the concentration of osmolyte are known from literature for the 70k dextran [9], the PEG 20k [24], and the PEO 200k [5] polymer solutions. Using the MATHEMATICA function NonlinearModelFit, the dominant-mode model is fitted to the volumetric measurements (see Figs. 1-3). In case the fit routine did not converge, the value of $K$ or $\kappa$ was fixed from the estimated value determined with, respectively, $\mathrm{CM}$ or a conventional permeability test [5] (see Tables I and II for a summary of, respectively, the fit parameters for the microgel and the aquapearl particles).

As explained in Sec. III B, the dominant-mode model contains the effective values for the material parameters $K, \kappa$, $D_{\text {osm }}$, and $\Pi_{\text {exc }}$. Nevertheless, we do expect the fitted values to give the typical order of magnitude and trends in the material parameters, e.g., the magnitude of the solvent quality pressure $\Pi_{\text {exc }}$ is expected to increase with osmolyte concentration. For the microgels, the order of magnitude of the compressive modulus $K$ agrees with independent measurements from CM [4], where the compressive modulus of the medium 
TABLE II. Material properties based on the model fits displayed in Figs. 2 and 3. The upper and lower sections of the table display the swelling and compression cases, respectively. The standard error for each derived material parameter is given in brackets. We assumed all fitted parameters to be uncorrelated.

\begin{tabular}{lcccc}
\hline \hline Solution & $\begin{array}{c}K \\
(\mathrm{kPa})\end{array}$ & $\begin{array}{c}\kappa \\
\left(\mathrm{nm}^{2}\right)\end{array}$ & $\begin{array}{c}D_{\mathrm{osm}} \\
\left(\mu \mathrm{m}^{2} / \mathrm{s}\right)\end{array}$ & $\begin{array}{c}\Pi_{\mathrm{exc}} \\
(\mathrm{kPa})\end{array}$ \\
\hline Saline & 27() & $7.5(0.5)$ & $0.0(0)$ & $0.0(0)$ \\
Dextran 70k 8 wt\% & $35(5)$ & $5.5(1.0)$ & $1.0(16.1)$ & $-6.8(41.5)$ \\
PEG 20k 8 wt\% & $86(8)$ & $2.2(0.5)$ & $1.7(36.8)$ & $-46.3(297.0)$ \\
PEO 200k 2 wt\% & 27() & $7.9(0.5)$ & $45.1(53.8)$ & $-17.6(4.2)$ \\
PEO 200k 4 wt\% & 27() & $7.6(0.5)$ & $82.1(35.1)$ & $-38.6(3.6)$ \\
PEO 200k 5 wt\% & 27() & $6.9(0.8)$ & $62.4(28.3)$ & $-49.7(4.3)$ \\
PEO 200k 7.5 wt\% & $31(2)$ & $7.5(0)$ & $19.5(29.6)$ & $-48.4(9.9)$ \\
PEO 200k 10 wt\% & $43(2)$ & $7.5(0)$ & $5.5(14.6)$ & $-79.6(22.1)$ \\
Dextran 70k 8 wt\% & $37(1)$ & $10.5(0.6)$ & $6.4(1.8)$ & $-4.0(0.5)$ \\
PEG 20k 8 wt\% & $75(1)$ & $5.3(0.2)$ & $3.9(4.8)$ & $-46.2(11.3)$ \\
PEO 200k 4 wt\% & 27() & $25.4(1.1)$ & $81.6(3.6)$ & $-40.7(0.5)$ \\
PEO 200k 7.5 wt\% & $153(10)$ & $16.3(1.4)$ & $78.2(4.4)$ & $-315.0(25.4)$ \\
PEO 200k 10 wt\% & $219(16)$ & $14.4(1.3)$ & $109.0(7.0)$ & $-562.0(49.0)$ \\
\hline \hline
\end{tabular}

particles was found as $K=13(5) \mathrm{kPa}$, with the uncertainty in brackets. The permeability $\kappa$ decreases with increasing bulk modulus, which is to be expected, as a higher bulk modulus implies a smaller cross-linker to cross-linker distance and therefore a smaller mesh size and permeability. The odd one out, however, is the PEO $2 \mathrm{M}$ experiment on soft particles, which disagrees with the $13-w t-\%$ dextran $70 \mathrm{k}$ experiment on the compressive bulk modulus of the soft particles. In this experiment we chose to set $D_{\text {osm }}$ and $\Pi_{\text {exc }}$, which given the large size of the osmolyte are expected to be very small, to zero in order to improve the convergence of the fit.

The osmolyte diffusion constant inside the hydrogel is generally expected to be a fraction of its value in the surrounding polymer solution [25]. Indeed, the fitted values for $D_{\text {osm }}$ are a fraction of the mutual diffusion coefficient in a $13-w t-\%$ dextran 70k solution, $D_{\mathrm{osm}}=6 \times 10^{1} \mu \mathrm{m}^{2} \mathrm{~s}^{-1}$ [20]. Finally, the solvent quality pressure $\Pi_{\mathrm{exc}}$ increases with the concentration of osmolyte, as expected.

For the aquapearl swelling experiments the fitted permeability agrees with independent measurements, which indicate $\kappa=8(5) \mathrm{nm}^{2}$ [5]. Moreover, the compressive bulk modulus agrees to that obtained by CM [5], except for the PEG 20k swelling experiment. The osmolyte diffusion coefficients all have a rather large uncertainty. For the PEO 200k experiments this is probably caused by polydispersity in osmolyte size [5] (see below). For the dextran 70k and PEG 20k swelling experiments the uncertainty may be connected to the fact that they do not exhibit an overshoot, thereby making the effect of osmolyte diffusion less pronounced. Finally, the PEO 200k swelling experiments show that the magnitude of the solvent quality pressure increases with the osmotic pressure, as expected.

The compression experiments on dextran 70k and PEG $20 \mathrm{k}$ agree with the appurtenant swelling experiments, showing the robustness of the dominant-mode model. The PEO 200k compression experiments, however, show anomalously high values for all four fit parameters. In Aangenendt et al. [5],
TABLE III. Fitted material properties from the PEO 200k 7.5-wt- $\%$ experiment where the data points are weighted proportional to their time coordinate (see the dashed line in Fig. 3). The extracted diffusion coefficient corresponds, most probably, to a high molecular weight fraction of the osmolyte.

\begin{tabular}{lcccc}
\hline \hline Solution & $\begin{array}{c}K \\
(\mathrm{kPa})\end{array}$ & $\begin{array}{c}\kappa \\
\left(\mathrm{nm}^{2}\right)\end{array}$ & $\begin{array}{c}D_{\mathrm{osm}} \\
\left(\mu \mathrm{m}^{2} / \mathrm{s}\right)\end{array}$ & $\begin{array}{c}\Pi_{\mathrm{exc}} \\
(\mathrm{kPa})\end{array}$ \\
\hline PEO 200k 7.5 wt\% & $40(1)$ & $3.2(0.2)$ & $1.6(0.4)$ & $-41(1)$ \\
\hline \hline
\end{tabular}

it was established that the PEO 200k osmolyte consists of a wide range of species having different sizes. As the different species have different diffusion constants and, possibly, different effects on the solvent quality, a complicated evolution of the volume of the hydrogel particle is to be expected. The dominant-mode model assumes only a single size of osmolyte molecules, however, implying the fitted material parameters to reflect a kind of average effect of all osmolyte species. As a result, the solid line fits in Fig. 3(a) show, for example, that the slow deswelling for $t \gtrsim 10^{5} \mathrm{~s}$ is effectively ignored, for the model fit equilibrates at $t \sim 10^{5} \mathrm{~s}$. Indeed, if we force the fit routine to take into account the final deswelling process by weighing each data point proportionally to the time coordinate, and hence giving more weight to the long-time data, the fit of, for example, the 7.5-wt-\% PEO 200k compression experiment provides credible values for all four fit parameters [see the dashed line in Fig. 3(a) and Table III for the fit parameters]. The fitted diffusion constant, most probably, pertains to a high molecular weight species of PEO $200 \mathrm{k}$, the solvent quality pressure agrees with the appurtenant swelling experiment, and the bulk modulus and permeability of the hydrogel particle agree with their independent measurement introduced above [5]. Moreover, the polydispersity in the PEO 200k osmolyte probably causes the large value and uncertainty of the fitted diffusion constant for the PEO 200k swelling experiments. The low molecular weight species cause a fast decrease in solvent quality, giving rise to the overshoot in hydrogel volume [see Fig. 2(a)].

\section{CONCLUSIONS}

Using the theory of poroelasticity we formulated a dominant-mode model which accounts for the swelling and compression of hydrogels in concentrated polymer solutions. The dominant-mode model is derived through a dominant-mode assumption: the long-time hydrogel dynamics in concentrated polymer solutions is assumed to be similar to that in dilute polymer solutions.

The equilibrium volume of the hydrogel is shown to be determined by the balance between the osmotic compressive force and the solvent quality pressure at the hydrogel surface. Diffusion of osmolyte results in nonmonotonic changes in hydrogel volume. On the one hand, osmolyte diffusion causes a gradual decrease in compressive force on the hydrogel particle, resulting in initial compression and subsequent reswelling. On the other hand, depending on whether the osmolyte increases or decreases the solvent quality for the hydrogel network, it can cause swelling or compression at 
the time scale of osmolyte diffusion. This paper shows that the spatiotemporal effect of osmolyte diffusion may cause nonmonotonic evolution of the hydrogel volume. In a previous relaxational dynamics model [5], osmolyte diffusion was treated phenomenologically, where only temporal feedback between osmolyte diffusion and hydrogel swelling was taken into account. Interestingly, nonmonotonicity was still observed, showing the temporal effect of osmolyte diffusion to be crucial to understand nonmonotonic (de)swelling of hydrogels.

Comparison of the dominant-mode model to experiment provides reasonable estimations of the compressive bulk modulus $K$ and the permeability $\kappa$ of the hydrogel network, the osmolyte diffusion constant, and the solvent quality change due to the presence of osmolyte. Therefore, the dominantmode assumption, which assumes the long-time hydrogel dynamics in dilute and concentrated polymer solutions to be similar, seems justified. One could argue that this is to be expected, since the material parameters do not vary strongly during the evolution in hydrogel volume. What is unexpected, however, is the fact that the dominant-mode model also accurately describes the short-time response of the microgel particles (see Fig. 1). We hypothesize this to be due to a cancellation of errors between the idealized description of the experimental setup and neglect of higher order modes in the exact solution.
In this paper we focused on osmolyte diffusion and the appurtenant effects of its osmotic pressure on the swelling and compression of hydrogels. The model can be extended to account for changes in the external $p \mathrm{H}$ and temperature; however, their spatiotemporal propagation is also governed by a diffusion equation. Therefore, we expect the dominant-mode model to have wider applicability. Finally, it is apposite to ask to what extent the implicit assumption of near-equilibrium made in our paper is justified beyond the fact that it appears to provide an adequate description, or, a fortiori, under what external conditions, e.g., magnitude of osmotic shocks, we can explicitly expect it to break down. This could be an interesting avenue of further research.

\section{ACKNOWLEDGMENTS}

We would like to acknowledge Paul van der Schoot for helpful discussions. The work of M.T.J.J.M.P. is part of the Industrial Partnership Programme Hybrid Soft Materials that is carried out under an agreement between Unilever Research and Development plc. and financed by the Dutch Research Council (NWO). The work of H.M.W. forms part of the research program of the Dutch Polymer Institute, Project No. 738; we are grateful for its financial support. The work of B.M.M. is part of the Dutch Research Council (NWO) and was performed at the research institute AMOLF.
[1] L. R. Kesselman, S. Shinwary, P. R. Selvaganapathy, and T. Hoare, Small 8, 1092 (2012).

[2] L. Ionov, Mater. Today 17, 494 (2014).

[3] S. J. Buwalda, K. W. Boere, P. J. Dijkstra, J. Feijen, T. Vermonden, and W. E. Hennink, J. Controll. Rel. 190, 254 (2014).

[4] J. J. Sleeboom, P. Voudouris, M. T. Punter, F. J. Aangenendt, D. Florea, P. Van Der Schoot, and H. M. Wyss, Phys. Rev. Lett. 119, 098001 (2017).

[5] F. J. Aangenendt, M. T. J. J. M. Punter, B. M. Mulder, P. van der Schoot, and H. M. Wyss, Phys. Rev. E 102, 062606 (2020).

[6] T. Tanaka and D. J. Fillmore, J. Chem. Phys. 70, 1214 (1979).

[7] M. Doi, J. Phys. Soc. Jpn. 78, 052001 (2009).

[8] H. M. Wyss, T. Franke, E. Mele, and D. A. Weitz, Soft Matter 6, 4550 (2010).

[9] C. Bonnet-Gonnet, L. Belloni, and B. Cabane, Langmuir 10, 4012 (1994)

[10] K. Devanand and J. C. Selser, Macromolecules 24, 5943 (1991).

[11] O. Coussy, Poromechanics (Wiley, New York, 2004).

[12] C. W. Macminn, E. R. Dufresne, and J. S. Wettlaufer, Phys. Rev. Appl. 5, 044020 (2016).

[13] V. Klika, Crit. Rev. Solid State Mater. Sci., 39, 154 (2014).
[14] R. L. Mauck, C. T. Hung, and G. A. Ateshian, J. Biomech. Eng. 125, 602 (2003).

[15] R. de Boer, Theory of Porous Media, 1st ed. (Springer-Verlag, Berlin, 2000).

[16] S. Peppin, J. Elliott, and M. Worster, Phys. Fluids 17, 053301 (2005).

[17] T. M. Quinn and A. J. Grodzinsky, Macromolecules 26, 4332 (1993).

[18] J. Tong and J. L. Anderson, Biophys. J. 70, 1505 (1996).

[19] M. N. Ozisik, Boundary Value Problems of Heat Conduction, Dover Books on Engineering (Dover, New York, 2013).

[20] B. N. Preston, W. D. Comper, A. E. Hughes, I. Snook, and W. van Megen, J. Chem. Soc., Faraday Trans. 1: Phys. Chem. Condens. Phases 78, 1209 (1982).

[21] T. Boudou, J. Ohayon, C. Picart, and P. Tracqui, Biorheology 43, 721 (2006).

[22] M. J. Krafcik and K. A. Erk, Mater. Struct. 49, 4765 (2016).

[23] J. C. Williams, L. A. Mark, and S. Eichholtz, Biophys. J. 75, 493 (1998).

[24] Harries Research Group, Osmotic stress data PEG 20k, https:// scholars.huji.ac.il/danielharries/book/peg-20k (accessed 12 December 2019).

[25] D. Sandrin, D. Wagner, C. E. Sitta, R. Thoma, S. Felekyan, H. E. Hermes, C. Janiak, N. De Sousa Amadeu, R. Kühnemuth, H. Löwen et al., Phys. Chem. Chem. Phys. 18, 12860 (2016). 